\title{
INTELIGENCIA ORGANIZACIONAL: APROXIMACIÓN TEÓRICA A SU CONCEPCIÓN Y DESARROLLO
}

\author{
INTELIGÊNCIA ORGANIZACIONAL: ABORDAGEM \\ TEÓRICA À SUA CONCEPÇÃO E DESENVOLVIMENTO
}

\author{
Sailys Uria López a \\ Yunier Rodríguez Cruz
}

\begin{abstract}
RESUMEN
Introducción: La Inteligencia Organziacional (IO) constituye una capacidad organizacional que sirve de apoyo a la toma de decisiones estratégicas. No obstante, la diversidad de términos y prácticas asociadas, ha incidido en la aplicación de múltiples enfoques con discímiles componentes, procesos y factores institucionales. Objetivo: En correspoondencia, este estudo pretende identificar y esclareces las bases teóricas y metodológicas que han incidido en el desarrollo de la $\mathrm{IO}$ y sus procesos particulares. Metodología: Se utiliza el Análisis Documental como principal métodos de investigación, y al análisis de contenidos permite identificar los principios y enfoques teóricos que han caracterizado esta capacidad. Resultados: A partir del análisis de los modelos y metodologías de 10 se identifican los componentes específicos de esta capacidad, su articulación con los procesos particulares de desarrollo: Vigilancia Tecnológica, Inteligencia Competitiva, Inteligencia Empresarial, Monitoreo de Información, así como los diversos elementos y factores a tener en cuenta para su desarrollo en las organizaciones. Conclusiones: La IO constituye una capacidad organizacional que se desarrolla a partir de un conjunto de procesos específicos y elementos particulares que sin su adecuada articulación, no se pueden obtener ventajas estratégicas y certeros procesos de decisión organziacional.
\end{abstract}

Descriptores: Inteligencia organizacional. Información. Conocimiento. Inteligencia. Organizaciones.

\footnotetext{
a Licenciada en Ciencias de la Información. Especialista en temas de Gestión de Información e Inteligencia Organizacional. E-mail: sai.uria0322@gmail.com

b Docente de la Facultad de Comunicación de la Universidad de La Habana. E-mail: yunier@fcom.uh.cu
} 


\section{INTRODUCCIÓN}

Las organizaciones del siglo XXI deben ser centros de innovación y aprendizaje, con estructuras de dirección lineales y descentralizadas, con visiones estratégicas y competitivas, con grandes flujos informacionales y proyectos de desarrollo de impacto socio-económico. Estas características se asocian al valor estratégico de la información, la que se ha convertido en un recurso esencial para el adecuado y efectivo desempeño organizacional. Desde los niveles operativos hasta el nivel estratégico organizacional, se observan flujos informacionales que permiten una efectiva toma de decisiones cuyos beneficios se relacionan con la creación de capacidades de sostenibilidad y competitividad. Ante estos nuevos enfoques y realidades contextuales del ámbito organizacional se ha consolidado en los últimos años la denominada Inteligencia Organizacional (IO).

El término "inteligencia" se ha usado, desde la antigüedad, como una capacidad de los individuos para resolver problemas que implican su intervención. Su origen, del latín "intelligentīa" alude a la capacidad de escoger o seleccionar las mejores opciones para solucionar determinado fenómeno. En correspondencia, la inteligencia es la capacidad que tiene el ser humano para asimilar, pensar, elegir y ejecutar una decisión para la resolución de un problema. Desde un análisis etimológico se entiende que la palabra inteligencia tiene que ver directamente con la capacidad intelectual desarrollada por los seres humanos para tomar una decisión o como la capacidad de crear productos con posibilidades de tomar determinado valor para la sociedad o para las organizaciones.

La Inteligencia Organizacional (IO), por su parte, encuentra su génesis en la propuesta de Sun Tzu en el libro "El arte de la guerra", el cual no se hizo popular únicamente por las estrategias militares que proponía, sino además porque sus lecciones podían ser aplicadas a diversas áreas como la de la administración de empresas. Por este motivo el libro ha ganado gran aceptación entre los líderes políticos y empresariales, incidiendo en la administración pública y la planificación. 
Cuando una organización se comienza a preocupar por sus resultados, por elevar los estándares de calidad de sus productos y servicios, y en consecuencia ser más eficaz y eficiente, esta requiere de un uso intensivo de información y del conocimiento. El uso de estos activos organizacionales se potencia a partir de procesos gerenciales como la Gestión Documental, de Información y del Conocimiento, pero la interacción entre estos recursos se puede optimizar bajo un enfoque integrado desarrollado bajo la IO.

El objetivo de esta investigación es identificar los componentes, factores organizacionales y características de la IO necesarias para que una organización sea eficaz, eficiente y efectiva en su gestión. La consulta de fuentes documentales y no documentales ha sido un punto de partida para el desarrollo de esta investigación. El análisis documental ha permitido conocer el estado del arte del tema, así como las posturas de los principales autores del tema.

\section{INTELIGENCIA ORGANIZACIONAL: ORIGEN Y EVOLUCIÓN EN LOS CONTEXTOS ORGANIZACIONALES}

En la literatura especializada en temas de inteligencia, se percibe un incremento de investigaciones empíricas y teóricas a partir de la década del '90 del pasado siglo XX, que han intentado conceptualizar y generar conceptos en relación a este término y su implementación en los contextos organizacionales. El análisis conceptual sobre la Inteligencia Organizacional (IO) permite identificar elementos y componentes claves para su concepción y desarrollo.

Son varios los autores que han definido la 10 en los contextos organizacionales, entre ellos se encuentran Wilensky (1967), Matsuda (1992), Halal (1998), Choo (1999), Haeckel y Nolan (1993), McMaster (1996), Oberschulte (1996), Cubillo (1997), Orozco Silva (1999), Núñez-Paula (2002), Albrecht (2002), North y Pöschl (2003), Más-Basnuevo (2005b), Akgün, Byrne y Keskin (2007), Yaghoubi, Gholami y Armesh (2012), Kiani, PourjafariJozam y PourjafariJozam (2013), Lozano-Oviedo y González-Campo (2013), Haber-Vega (2015), González (2015), Teixeira y Valentim (2015) y Rodríguez-Cruz (2015).

A pesar de que el término se patenta en 1967, no fue hasta el 1992, en una Conferencia Internacional sobre Economía en Tokio, Japón, que Takehiko 
Matsuda presenta un artículo titulado "Inteligencia Organizacional: su importancia como proceso y como producto" que tuvo gran impacto en la comunidad científica, es entonces cuando se comienza a prestarle atención a este fenómeno. Matsuda (1992) considera que "La IO puede ser vista como un proceso o como un producto y está compuesta por varios procesos: percepción, almacenamiento, aprendizaje, comunicación y decisión." En este sentido, señala dos componentes interdependientes: la IO como proceso (dinámico) y la IO como producto (estático).

Wilensky (1967), fue el primero en utilizar el término inteligencia organizacional (IO) y en su definición resalta la importancia estratégica del mismo al concebirlo como el acto de "[...] reunir, procesar, interpretar y comunicar la información que se necesita en el proceso de tomar decisiones".

Halal (1998) entiende que la IO es "la capacidad de una organización para crear conocimiento y usarlo para adaptarse estratégicamente a su ambiente". En este sentido, se puede mencionar que para Halal las organizaciones tienen su propio coeficiente intelectual proveniente de los recursos humanos que emplea, y de los sistemas de tecnología inteligente usados para comprender redes de información complejas dentro de la institución.

Choo (1999), por su parte, expone que

[...] es la propiedad que emerge de la red de procesos de uso de información mediante la cual la organización construye significados compartidos acerca de su identidad y actividad; descubre, comparte y aplica nuevo conocimiento, e inicia patrones de acción mediante la búsqueda, la evaluación y la selección de opciones.

Se considera que Choo (1999) entiende la IO como propiedad a la capacidad de las organizaciones para analizar y comprender las situaciones reales que forman parte su ambiente (interno o externo) a partir de la deconstrucción de contenidos informacionales que pueden abonar nuevos conocimientos que sean de valor significativo para tomar decisiones.

De acuerdo con Choo (1999), Haber-Vega (2015) plantea la necesidad de IO para el apoyo a la toma de decisiones acertadas "La inteligencia como capacidad que le permite a la organización adaptarse al ambiente externo; la 
creación y uso del conocimiento como procesos que favorecen la formación y desarrollo de la IO y la toma de decisiones estratégicas."

Los supuestos de Más-Basnuevo (2005 ab) y North y Pöschl (2003) suman, aunque en distinto modo, procesos de apoyo que sustentan la IO y que son necesarios para el funcionamiento eficaz de las organizaciones como la capacidad de resolución de problemas, la planificación, el análisis y la evaluación.

Müller y Castillo (2012) exponen que la IO es una "Disciplina capaz de ayudar a las organizaciones en la integración de sus procesos, colaboradores y tecnologías, contribuyendo para una comprensión de una empresa, como un sistema abierto que necesita de una constante observación de sus ambientes."

Kiani, PourjafariJozam y PourjafariJozam (2013) consideran que "la 10 tiene como consecuencias la innovación, ventajas competitivas, mejor desempeño y eficiencia, se puede concluir que esas consecuencias y resultados puedes ayudar a las organizaciones a adaptarse al ambiente y sus cambios y finalmente sobrevivir." De acuerdo con esto, Lozano-Oviedo y González-Campo (2013) observan que la 10 es "Capacidad imprescindible para que las organizaciones se sostengan con éxito a través del tiempo, requiere de la gestión permanente de los distintos componentes que la constituyen."

Müller y Castillo (2012); Kiani, PourjafariJozam y PourjafariJozam (2013) y Lozano-Oviedo y González-Campo (2013) señalan en sus definiciones las fronteras de la organización como su salida al mundo. Además observan procesos, tales como la innovación, que ocurren al interior de la organización y que se traducen en eficiencia y eficacia de los productos/servicios que ofrece la institución.

Aunque para algunos autores como Matsuda (1992), González (2015) y Teixeira y Valentim (2015) la IO es vista como un proceso; se observa que Haeckel y Nolan (1993), McMaster (1996), Oberschulte (1996), Cubillo (1997), Halal (1998), Orozco Silva (1999), Choo (1999), Núñez-Paula (2002), Albrecht (2002), North y Pöschl (2003), Más-Basnuevo (2005b), Akgün, Byrne y Keskin (2007), Yaghoubi, Gholami y Armesh (2012), Kiani, PourjafariJozam y PourjafariJozam (2013), Lozano-Oviedo y González-Campo (2013), Haber-Vega 
(2015) y Rodríguez-Cruz (2015) coinciden en ver la IO como una capacidad de las organizaciones para la solución de problemas dentro de una organización y la facilitación del proceso de toma de decisiones de los directivos. En este sentido, el tratamiento de la IO por parte de la institución donde se aplica, debe contar con el apoyo del nivel estratégico; Teixeira y Valentim (2015) apuntan a una visión de 10 como proceso estratégico, por lo cual quienes tienen el deber de tomar decisiones deben ser los primeros en abogar por tener una organización inteligente y promover programas de identidad corporativa que impulsen una cultura y el clima organizacional adecuado; además si un líder se muestra interesado por implantar la 10 destinará tiempo, recursos y esfuerzos para conseguir su objetivo.

Desde el punto de vista conceptual hay que destacar que, en las definiciones analizadas, se observa el uso de términos que implican una capacidad intelectual y analítica elevada, entre ellos aprender, comprender, analizar, resolver, modelar, implementar, asimilar e integrar. Es por ello que se puede ver la IO como una capacidad, pues los procesos que la propia IO engloba le dan un carácter más complejo e intelectual y menos técnico y procesual.

Una vez examinadas las principales definiciones sobre la IO, se puede referir que la Inteligencia Organizacional constituye una capacidad institucional que permite desarrollar adecuados procesos cognitivos e informacionales, de forma que las organizaciones puedan -bajo un enfoque de aprendizaje continuopercibir y analizar información de forma estratégica, generar conocimiento organizacional y tomar decisiones efectivas para solucionar problemas y aprovechar oportunidades, tener un mejor desempeño organizacional y alcanzar un buen posicionamiento organizacional de manera más eficaz y eficiente.

\section{LA INTELIGENCIA: NOCIONES Y POLÉMICAS CONCEPTUALES}

En la literatura especializada sobre el tema de IO, se conciben múltiples conceptos y procesos que si bien se relacionan, también se distinguen por presentar características particulares con objetivos y propósitos bien definidos. Los mismos, sin embargo, deben y pueden visualizarse dentro de la 10 como capacidad organizacional que garantiza su acertado desarrollo. 
Los temas relacionados a la 10 son emergentes y es muy común encontrarse con autores que tienen diferentes visiones en el momento de nombrar el tipo de inteligencia a la que aluden. En consonancia con esto y en aras de discernir este fenómeno se han estudiado las propuestas de diferentes teóricos sobre el tema.

Haber-Vega y Más-Basnuevo (2013) plantean que los anglosajones encuentran sinonimia entre las categorías Inteligencia Organizacional (IO) e Inteligencia Empresarial (IE), mientras los españoles entienden que Inteligencia Corporativa e Inteligencia Competitiva son concepciones semejantes. En el discurso de estas autoras afirman que la 10 es la más amplia; en este sentido, las autoras mencionan que "La 10 incluye la combinación del paradigma cognitivo con el paradigma sociocultural para desarrollar la capacidad de aprendizaje continuo a todos los niveles organizacionales."

Tyson (1998) define la IE como

[...] un proceso analítico que transforma datos en conocimiento estratégico relevante y preciso, donde se conoce la información sobre la posición de los competidores, su desempeño, capacidad e intenciones, sobre las fuerzas motoras del mercado, sobre productos y tecnologías específicas.

Ponjuán-Dante (1998) define que la

[...] inteligencia corporativa es la capacidad y la función de reunir, analizar y diseminar datos que permiten obtener, de manera sistemática y organizada, información relevante sobre el ambiente externo y las condiciones internas de la organización, para la toma de decisiones y la orientación estratégica.

Orozco Silva (2009), plantea que la IE es "la capacidad de reunir, analizar y diseminar datos, lo que permite obtener, de manera sistemática y organizada, información relevante sobre el ambiente externo y las condiciones internas de la organización, para la toma de decisiones y la orientación estratégica." Se puede observar que las percepciones de este autor cambian: ya no solo se refiere a la IE como la obtención de la información (OROZCO SILVA, 2001), sino que hace alusión a otros procesos intelectuales y para ello se acoge a la cita de PonjuánDante (1998) sobre inteligencia corporativa.

Al referirse a la Inteligencia Competitiva (IC), Da Silva (2015) sugiere que "es una capacidad para la innovación, donde los recursos dependen en gran medida del conocimiento individual y organizacional." 
Se puede apuntar que tanto Orozco Silva (1999) y Da Silva (2015) concuerdan en que la IC se limita a la obtención de datos en el entorno organizacional (interno y externo) que luego transforma en conocimiento estratégico relevante y preciso y en un producto que facilita la toma de decisiones.

Según lo planteado por Haber-Vega (2015) "la IC y la IE constituyen herramientas gerenciales; la IO es además, un instrumento cultural, una forma de hacer a todos los niveles de la organización."

Núñez-Paula (2002) declara que

[...] no debería confundirse el concepto amplio de Inteligencia Organizacional, o incluso los genéricos Inteligencia Corporativa o Empresarial con sus sistemas de procesos de gestión, más específicos, que contribuyen a mantener la vigilancia sobre diversos aspectos del entorno de la organización como son: Vigilancia (de diferente tipo según sus objetos), Monitoreo, Inteligencia Competitiva, Comercial, de Negocios, Tecnológica, etc.

Es válido aclarar que para estos autores la 10 obviamente encuentra puntos comunes con la IE y la IC y eso se debe a que la IO es superior a las particularidades de cada uno de estos tipos de inteligencia. Sin embargo, en aras de diferenciar y marcar los límites entre una y otra los autores de esta investigación se apoyan en la Figura I.

Figura 1 - Diferencias entre IO-IE-IC-Inteligencia corporativa

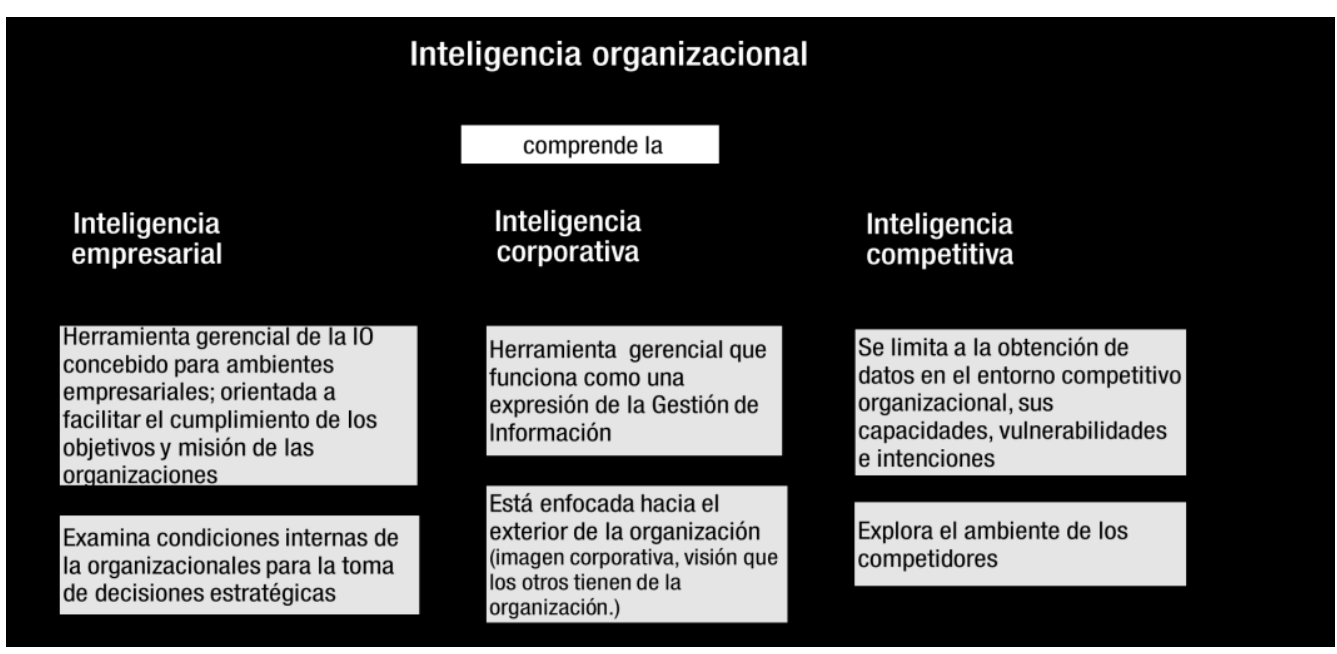

Fuente: Elaboración propia.

Entre los elementos comunes de la IO-IE-IC se identifican: 
- $\quad$ Enuncian la información y el conocimiento como recursos clave de la organización.

Reconocen la importancia de la gestión de la información y el conocimiento para la efectiva toma de decisiones y la orientación estratégica.

Relacionan la inteligencia con el desempeño organizacional de acuerdo con su alcance, contexto y fines.

- Sus procesos contribuyen a la organización, pues sus tareas redundan en procesos de gestión de información, gestión del conocimiento y de vigilancia.

Se coincide con las ideas de Orozco Silva (1999), Núñez-Paula (2002), Más-Basnuevo (2005a), Haber-Vega (2015) y Rodríguez-Cruz (2015) pues todos aluden que la IO debe ser calificada como el concepto más general, al interpretar esta capacidad en el contexto de cualquier tipo de organizaciones. De esta forma la IO es un concepto que enuncia una capacidad organizacional que incide directamente en la creación de valor estratégico. Esta capacidad genera a su vez procesos y sistemas particulares de vigilancia tecnológica, inteligencia competitiva, monitoreo informacional $u$ otros tipos de inteligencia en las organizaciones.

\section{CARACTERÍSTICAS DE LA INTELIGENCIA ORGANIZACIONAL}

Las organizaciones se visualizaban como sistemas estables, cerrados, con limitadas interacciones con su contexto, y con poca o ninguna actividad innovadora. Esta visión se ha modificado sustancialmente en los últimos años con el uso del término "organizaciones inteligentes". Este último alude a organizaciones flexibles y dinámicas, abiertas en su relación e interacción con su contexto, con gran capacidad innovadora ya que desarrollan procesos de percepción, aprendizaje organizacional, creación de conocimiento y una efectiva toma de decisiones. A consideración de Choo (1999), este tipo de organizaciones se caracterizan por:

- "La organización que es capaz de integrar eficazmente la percepción, la creación del conocimiento y la toma de decisiones se puede describir como una organización inteligente. 
- La organización inteligente que es hábil en la creación, adquisición y transferencia de conocimiento y en la modificación de su conducta para relegar su nuevo conocimiento y pericia.

- La información y el conocimiento, le permiten maniobrar con inteligencia, creatividad y en ocasiones astucia.

- La organización inteligente está preparada para sostener su crecimiento y desarrollo en un ambiente dinámico, al percibir y comprender su entorno y adecuarlo a recibir oportunidades para una adaptación.

- Aplica reglas y rutinas para la toma de decisiones ya aprendidas.

- El manejo de los procesos de información, sustentan la percepción, la creación de conocimientos y la toma de decisiones.

- La organización inteligente es eficaz porque evoluciona continuamente junto con el entorno cambiante, renueva su arsenal de conocimientos y practica un atento procesamiento de información en su toma de decisiones.

- Mediada, ya que las interacciones que se producen entre los sistemas, individuos, rutinas u objetos son mediadas a través de reglas, roles, relaciones estructurales (formales e informales) y el uso de herramientas y de las tecnologías de información y comunicación.

- Situada, ya que se encuentra localizada en un tiempo y espacio, lo que le permite interactuar con los pormenores físicos y sociales donde se efectúa esta práctica.

- Provisional, ya que las rutinas, reglas, políticas y roles en los que se fundamenta, cambian en dependencia de los intereses de las organizaciones, así como las formas de conocer y hacer.

- Pragmática, ya que produce una acción que está enfocada en fines y que se dirige al objeto de la actividad. Esta acción depende de las concepciones que posea el personal de la organización del objeto de sus actividades.

- Disputada, ya que es vista con frecuencia como un recurso de poder, donde el desarrollo de actividades como la determinación del acceso a la información, la capacitación, la resolución de problemas, la toma de decisiones, etc. pueden generar conflictos."

Miller (2002) expone que entre las características de la inteligencia están: 
- Ofrecer información filtrada, depurada

- Proceso que acaba proporcionando oportunidades únicas relativas a las decisiones futuras que dan margen y ventajas sobre los competidores

Teixeira y Valentim (2015), por su parte, caracterizan a la IO como:

- Proceso que debe ser construido, mantenido y evaluado constantemente

- Proceso movida por una dinámica de búsqueda, compartimiento y uso de información de modo creativo para descubrir oportunidades, reducir riesgos y propiciar diferentes tipos de innovación combinadas, implicando más calidad, productividad y competitividad.

- Proceso estratégico con una estrecha relación con la gestión de información

- Ayuda a las organizaciones a crear valor agregado y estratégico, para garantizar las diferencias con los competidores

La IO se orienta a una adecuada proyección estratégica de la organización a corto, mediano y largo plazo, bajo la premisa de la satisfacción plena de las necesidades de los usuarios o clientes de la organización. De igual forma apuesta por los procesos de innovación bajo un aprendizaje continuo lo que favorece la competitividad institucional.

Estas características que identifican Choo (1999), Miller (2002), y Teixeira y Valentim (2015) permiten afirmar que la IO constituye una capacidad informacional que se centra en una toma de decisiones efectivas para la creación de valor estratégico, centrado mayoritariamente en las oportunidades organizacionales. Dicha capacidad se genera bajo adecuados procesos de percepción, creación de conocimiento y toma de decisiones a todos los niveles institucionales, bajo el reconocimiento de la información y el conocimiento como recursos y activos estratégicos. Esta capacidad genera mayor adaptabilidad ante cambios y oportunidades del ambiente externo a partir del desarrollo de procesos informacionales como la búsqueda y recuperación de información, el procesamiento y análisis de la misma. Es por ello que también la $\mathrm{IO}$ aboga por la Gestión del Cambio a través de determinadas capacidades dinámicas para la adaptación y el aprendizaje, constituyendo un proceso que tributa a la calidad, la 
productividad y la competitividad, en su estrecha relación entre la Gestión de Información y la Gestión del Conocimiento.

En este sentido, la IO está bajo la influencia de determinados factores o elementos organizacionales como: el poder y el liderazgo organizacional, las relaciones sociales y redes de trabajo institucional, el contexto real en el que se desenvuelve la institución, las normas, programas, reglas y rutinas organizacionales, así como la cultura informacional u organizacional que existe en la organización.

Haber-Vega y Más-Basnuevo (2013) consideran que "la IO es además, un instrumento cultural, una forma de hacer a todos los niveles de la organización." Esta característica es vital porque la cultura organizacional según Alabart y Portuondo (apud ALABART-PINO, 2003) es

[...] un conjunto de paradigmas, que se forman a lo largo de la vida de la organización como resultado de las interacciones entre sus miembros, de estos con las estructuras, estrategias, sistemas, procesos, y de la organización con su entorno, a partir de las cuales se conforma un conjunto de referencias, que serán válidas en la medida que garanticen la eficiencia, la eficacia y la efectividad de la organización.

Por su parte, más allá de caracterizar, Kiani, PourjafariJozam y PourjafariJozam (2013) expone una serie de requerimientos que deben estar contemplados en la infraestructura de una organización para ser inteligente, entre ellos:

- Gestión del capital intelectual en la organización

- Gestión del conocimiento

- Hacer cambios en la dirección

- Capital social

- Aprendizaje organizacional

- Cultura organizacional

- Estructura organizacional

- Gestión del talento

Cada uno de estos componentes de la organización desempeña un rol fundamental en el momento de implantar la IO, pues hacen de la organización una institución moderna, descentralizada, eficaz, dinámica, sistémica y, por sobre todas las cosas, enfocadas en desarrollar el aprendizaje continuo y el 
conocimiento en sus miembros.

Si bien se han podido precisar algunas características de la 10 , es necesario enfatizar que estas capacidades dinámicas que se asocian a la 10 se deben generar a partir de la visión y el autoreconocimiento de la organización. Esto es válido ya que depende de los esfuerzos y el empeño de los directivos en desarrollar estas capacidades específicas. Es por ello que lo importante resulta trazar los objetivos estratégicos que permitan transitar hacia la creación de la IO como capacidades y bajo el desarrollo de procesos puntuales de la inteligencia. De esta manera, las organizaciones inteligentes, van a desarrollar sus propias características a partir del contexto y las capacidades organizacionales para adaptarse a los cambios y la gestión de las competencias de los miembros de la organización.

\section{MODELOS Y METODOLOGÍAS DE INTELIGENCIA ORGANIZACIONAL}

(IO)

Un modelo es la representación abstracta, conceptual o gráfica de un fenómeno, sistema o proceso y se traza con el objetivo de analizar, interpretar o describir el comportamiento de este. La creación de modelos ha estado muy ligada al eje central de la actividad científica, pues a través de los modelos se pueden predecir los eventos futuros y resultados de la actividad en estudio. La creación de modelos de $\mathrm{IO}$ ha permitido identificar las principales etapas de este proceso como sistema dentro de una organización y ver los factores organizacionales a los que involucra.

El modelo de Halal (1998) se basa en medir la IO tal como se hace en los seres humanos. Propone un diagnóstico de lo que no está funcionando bien en la organización, por lo cual la propuesta de Halal es válida sobre todo para la identificación de los problemas que afectan el desarrollo total de la IO. El autor plantea que cualquier fallo en alguno de los subsistemas afecta a las Tecnologías de Información y Comunicación (TIC) de diversas maneras, porque cada subsistema contribuye a la IO y tiene un impacto en la organización.

North y Pöschl (2003) presentan un tipo de medición de inteligencia en las organizaciones a partir de test psicológicos cuyo resultado es expresado, al igual 
que en la inteligencia humana, en términos de coeficiente de inteligencia. Esto, aparejado con la técnica del benchmarking, facilita la capacidad de la organización para solucionar problemas de manera eficiente. La importancia de este modelo radica en su capacidad para integrarse con otros modelos. Un punto a favor en este modelo es que sus autores proponen una matriz para la autoevaluación de la organización que relaciona cinco dimensiones (Mercados/Competidores, Clientes/Proveedores, Productos, Procesos, y Empleados) que deben ser contrastadas con los componentes de su modelo. Esta matriz posibilita que las organizaciones conozcan cuidadosamente sus capacidades y competencias internas, así como las de sus competidores, para evaluar y medir su situación competitiva. Haber-Vega (2015) identifica una limitación en este modelo y es que "no se visualiza la relación de la cultura organizacional y su fomento con el proceso de introducción de la IO", sin embargo se considera que tanto la Memoria organizacional como la Inteligencia emocional están estrechamente relacionadas con los individuos y la cultura organizacional y, estas a su vez con la IO.

Por otra parte, el modelo de Choo (1999) contempla tres elementos principales: percepción, creación de conocimiento y toma de decisiones. Este autor asume los procesos principales del ciclo de vida de la $1 \mathrm{O}$ y los propone como la base para plantearse su modelo. Choo señala el uso estratégico de la información en las organizaciones para enfrentar los cambios del ambiente externo y facilitar el proceso de tomar decisiones, a partir de estos tres elementos. De este modo, hay espacio en su estudio para insertar el procesamiento de la información, como proceso imprescindible para convertir la información en un recurso susceptible de ser interpretado a partir de percepciones y con la cual se puede crear nuevo conocimiento y tomar decisiones en base a ello.

Se puede decir que el modelo de Choo (1999) aporta un carácter sistémico y relacional (pues se logran tejer redes a partir de la interpretación de significados), reconoce la existencia de marcos de referencias (desarrollados sobre la base de las experiencias pasadas y que son usados por los gerentes de una organización para calificar, tomar decisiones, desarrollar conductas de 
comunicación entre los miembros de un grupo que les permita ampliar sus ideas para dar cabida a múltiples interpretaciones), tiene un enfoque integrador (logra enlazar las etapas que propone en su ciclo de inteligencia con los procesos del modelo de (O) y logra discernir los subprocesos que forman parte de su modelo de IO y que tienen su raíz en análisis de la Teoría de organizaciones y en las Ciencias de la Información (destaca la interrelación de la gestión de documental, de información y del conocimiento, la gestión por procesos, y la cultura organizacional en el aprendizaje y la adaptación organizacional). Además, el modelo tiene un perfil social y dinámico, que muestra al individuo como ser perceptible y generador de conocimiento útil para la organización.

El modelo de Cronquist (2004), es una propuesta que abarca los principios esenciales que forman un sistema inteligente exitoso en la organización. Estos principios incluyen: atención, motivación, apoyo organizacional, suministro de información y calificaciones. El modelo aporta una visión evaluadora, hacia la calificación de los procesos informacionales y de inteligencia que tienen lugar dentro de la organización. Sin embargo, aunque hay coherencia entre los componentes de Cronquist, una de las limitaciones es que no hay un enfoque sistémico en el modelo, sino que los componentes son vistos como tareas independientes sin una relación clara entre ellos.

Estos principios para $1 \mathrm{O}$ se relacionan con los subsistemas de Halal (1998), pues la motivación de la que habla Cronquist es proporcional a la cultura y clima organizacional que propone Halal; con el modelo de Choo (1999), en cuanto a la calificación que recibe una información por su calidad para facilitar el proceso de toma de decisiones organizacionales; y con las variables del modelo IO de North y Pöschl (2003), ya que la fusión de los componentes de Cronquist contribuyen a la capacidad de respuesta y la capacidad para resolver problemas en una organización.

Más-Basnuevo (2005b) plantea que

[...] solo se puede implementar el modelo de IO si en una organización están presentes los siguientes principios: relación sistémica, efectividad de la formación y el desarrollo, participación activa y democrática, motivación, emprendimiento organizacional, autonomía, control, auto organización y aceptación de lo difuso. 
Dichos principios acompañados de los componentes del modelo que MásBasnuevo (2005b) propone: "[...] los individuos y grupos, los procesos en los que estos participan, los métodos y medios que utilizan para ejecutar los procesos y relacionarse y las propias relaciones interpersonales, intergrupales dentro de la organización o interorganizacionales" posibilitan que se dinamicen los procesos y que se cumpla la estructura propuesta. La autora nombra a su modelo Espiral de IO y lo hace porque intenta la combinación del paradigma cognitivo (se apoya en las habilidades de las personas para desarrollar las funciones de gestión de información y del conocimiento) y del paradigma sociocultural (que está mediado por sentimientos, voluntades personales, tradiciones, etc.) para desarrollar la capacidad del aprendizaje continuo.

El modelo de Más-Basnuevo aporta una visión estratégica e integradora de las funciones organizacionales. Al contrario de otros modelos, no solo valora los procesos que posibilitan la IO; además, añade un enfoque de agregación de valor que indica que a medida que ocurren procesos que transforman la información en recurso el valor aumenta, según se observa en la representación.

Haber-Vega (2015) realiza un estudio de los modelos de IO propuesto por varios autores y de las metodologías que otros desarrollaron en aras de alcanzar la IO. Tomando como plataforma el concepto y el modelo de IO enunciado por Más-Basnuevo (2005b), Haber-Vega desarrolla una metodología con un enfoque sistémico, donde cada salida constituye una próxima entrada. La metodología tiene tres etapas que responden a los procesos fundamentales que desarrolla, cada una de ellas tiene fases de ejecución y acciones que se deben realizar en busca de una mejor implantación. La primera consiste en un diagnóstico, la segunda etapa radica en la implantación de la lO y la tercera etapa se enfoca en la evaluación. La propuesta de Haber-Vega (2015) aporta un enfoque tecnicista y por procesos, pues da los pasos a seguir para la óptima ejecución de la metodología.

De manera general, los modelos y la metodología analizada coinciden en la segmentación de la $\mathrm{IO}$ a partir de procesos y fenómenos que tienen lugar en la organización. En este sentido, se puede decir que al integrar la suma de componentes por modelos se pueden establecer relaciones comunes entre ellos 
que indican el objetivo común que tienen: la formación de una organización inteligente a partir del desarrollo de ciertos componentes (Véase Figura II).

Figura 2 - Analogía entre los componentes de los modelos de 10 de autores estudiados

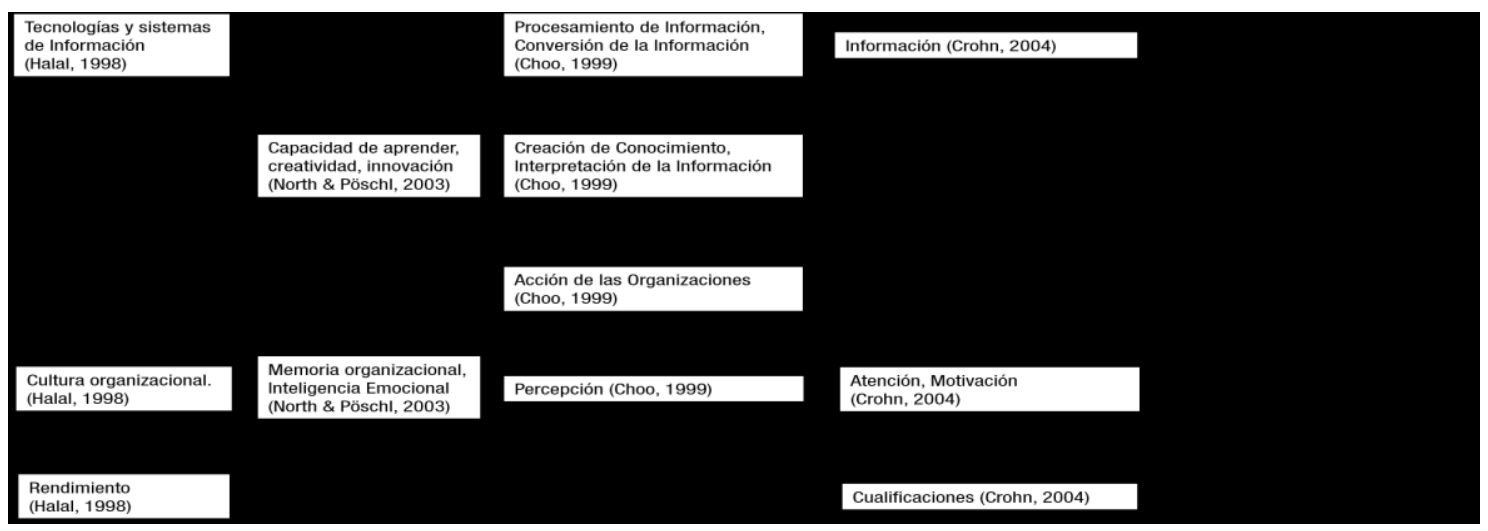

Fuente: Elaboración propia.

\section{PROCESOS, PRODUCTOS Y SERVICIOS ASOCIADOS A LA INTELIGENCIA ORGANIZACIONAL}

La IO puede tener varias miradas desde el mundo organizacional. Por sus funcionalidades puede ser entendida, según varios autores, como un proceso, un producto o un servicio; e incluso Alabart-Pino (2015) expone a "la inteligencia organizacional, [...] concebida como sistema, procesos o como función, dentro de la cultura y el funcionamiento organizacional."

Matsuda (1992) explica que la visión de 10 como proceso dinámico está conformado por cinco componentes: cognición organizacional, memoria organizacional, aprendizaje organizacional, comunicación organizacional y razonamiento organizacional. Los componentes propuestos por Matsuda (1992) son un soporte para otros procesos que tiene lugar en la organización. Sin embargo como producto considera que está conformado por datos, información e inteligencia.

La inteligencia en una organización puede ser definida como un proceso informacional y específicamente como un producto que requiere el uso de pautas colectivas para crear los sistemas de información organizacionales (ERFANIKHANGHAHIE; JAFARI, 2010). 
Según Orozco Silva (2009) "la IO brinda al mismo tiempo productos y servicios." Hay organizaciones, como las consultorías, que se dedican a prestar servicios a centros que requieren ayuda para desarrollar procesos de inteligencia y estos reciben el producto final de la inteligencia. Esto quiere decir que, dentro de la IO, la inteligencia competitiva (IC) pone énfasis en realizar productos de inteligencia sobre los competidores y en el monitoreo del medio, es por ello que se ve la IC como un producto y un proceso de la IO. Este tipo de inteligencia (IC) puede ser considerada como "un proceso (analítico), que transforma datos en conocimiento estratégico relevante y preciso, y un producto (la información ya procesada)" (OROZCO-SILVA, 2009).

Haber-Vega (2015) estudia la propuesta de varios autores y explicita que la 10

[...] tiene como procesos clave: la gestión documental, de información, del conocimiento y la toma de decisiones individuales, grupales y organizacionales; por su dependencia del adecuado acceso a la información y su uso, y de la creación, compartición y uso del conocimiento (procesos de apoyo); influenciada por factores como: la cultura y el clima organizacional, la gestión del capital humano, el desarrollo y utilización de las TIC, y la comunicación; que favorecen el aprendizaje organizacional, la innovación, la mejora continua, el aprovechamiento de oportunidades, y la creación de valor.

Es preciso destacar que, en la mayoría de los casos, los procesos de inteligencia competitiva que se desarrollan en las organizaciones son reconocidos en forma de sistemas. Sin embargo aunque estos subsistemas de la 10 contribuyen a su optimización, a ellos se suman otros como la cultura informacional para el desarrollo de los procesos informacionales en las organizaciones.

Las propuestas anteriores dejan claro que tanto como proceso, como producto, o como servicio la 10 tiene como eje central a la información y la importancia que tiene para las organizaciones que su flujo sea ininterrumpido, transparente y controlado de manera que permita elegir las mejores opciones para solucionar problemas del medio ambiente interno o externo a la organización.

Se puede identificar que la Inteligencia Organizacional constituye una capacidad institucional bajo la cual se desarrollan procesos y productos 
específicos asociados a la denominada Inteligencia Empresarial, Inteligencia Competitiva, Vigilancia Tecnológica o Monitoreo Informacional. De esta forma las capacidades generadas a partir de una buena búsqueda y selección de información, procesamiento y análisis para la toma de decisiones requiere el desarrollo de productos específicos a la medida de los directivos y eso se logra a partir de procedimientos y procesos particulares que se realizan bajo los preceptos de la inteligencia.

VI. Concepción de la Inteligencia Organizacional en contextos institucionales

Es indiscutible que para una institución de estos tiempos es de suma importancia convertirse en una organización inteligente, por las facilidades que esto representa para el flujo de procesos dentro de la organización. La IO no debe confundirse con la suma de la inteligencia de los individuos que laboran en la organización, sino que la IO constituye una capacidad organizacional bajo la cual pueden desarrollarse procesos, sistemas y productos relacionados con el monitoreo, la vigilancia, la IC entre otros tipos de inteligencia con un alcance más específico y delimitado. Lo cierto es que, sin dudas, una de las aplicaciones de la IO que más utilidad y beneficio trae a las organizaciones y sus directivos es la toma de decisiones acertadas. Es por ello que después de analizar los conceptos presentados y los modelos de los autores citados se deben puntear los elementos cognitivos e informacionales y factores organizacionales que van a definir el despliegue en las organizaciones.

Los elementos cognitivos e informacionales tales como el capital intelectual, memoria organizacional, capacidad para tomar decisiones, percepción, agregación de valor, aprendizaje organizacional, cognición organizacional, innovación, razonamiento organizacional, sistema de información, procesos informacionales, tecnologías de la información y la comunicación (TIC) ayudan al montaje de procesos de gestión del conocimiento y de la información en las organizaciones. La labor individual de cada uno de estos elementos no significa un aporte si no se ven de manera integrada las oportunidades de desarrollo que implica el funcionamiento como un todo de estos sistemas. La relación armónica de estos componentes se convierte en un 
todo superior que es la IO, lo cual demuestra su carácter holístico.

Entre los factores organizacionales que inciden en la $\mathrm{IO}$ se pueden citar la cultura organizacional, comunicación organizacional estructura organizacional, estrategia organizacional, ambiente interno y externo, clima organizacional, relaciones sociales, apoyo organizacional, motivación y recursos humanos. Cada uno de estos factores tiene un valor sistémico dentro de la organización, el estado en que se encuentren cada uno de ellos afecta al otro.

De ahí se deriva la necesidad de desarrollar esta capacidad a través del despliegue de sistemas, procesos, recursos y procedimientos particulares que garanticen crear condiciones y la infraestructura adecuada para generar organizaciones inteligentes.

De acuerdo con Choo (1999), la IO permite que las organizaciones puedan:

- Adaptarse de manera oportuna y eficaz a los cambios de su ambiente.

- Comprometerse en un aprendizaje continuo, que incluye desechar suposiciones, normas y estados mentales que ya no son válidos.

Haber-Vega (2015) refiere la importancia de desarrollar la IO "con el objetivo de fomentar la innovación, el aprovechamiento de oportunidades, el aprendizaje, y la toma de decisiones efectivas."

Como resultado del fuerte proceso de globalización, los cambios acelerados en el mercado y las ofertas de productos y servicios, la aparición de Internet y nuevas fuentes de información, la socialización de las investigaciones y sus aplicaciones prácticas, se ha propiciado que las organizaciones se encuentren en un ambiente cada vez más competitivo. Esto significa que: enfoques como la gestión de la información tengan un valor representativo como procesos gerenciales que actúan en función de determinar los elementos internos y externos que influyen en el cumplimiento de la misión y los objetivos de la organización y como colofón una organización más atemperada a la sociedad en la que se encuentra.

La competencia entre las organizaciones hace que estas alcancen mejores índices de desarrollo, aprendizaje e innovación, pues saben que tienen que sobrevivir al ambiente hostil que le crea la competencia. Esto no solo se 
aplica a organizaciones con fines de lucro, sino que cualquier organización es susceptible de tener una competencia declarada.

De esta forma, la concepción de la 10 en las organizaciones debe contemplar componentes determinados entre los que se encuentran los procesos informacionales de búsqueda, procesamiento y análisis de información para la toma de decisiones; el desarrollo de procesos y productos de inteligencia competitiva, vigilancia tecnológica y monitoreo informacional. Estos componentes se deben desplegar a todos los niveles de decisión (operativo, táctico, gerencial y estratégico) para el adecuado desarrollo de la Percepción Organizacional, Creación de Conocimiento y los procesos de decisión. El alcance de la IO se orienta a todo el contexto organizacional y busca fortalecerse a través de la integración de procesos gerenciales de apoyo como la Gestión Documental, la Gestión de Información y la Gestión del Conocimiento.

\section{CONCLUSIONES}

En 1967 se presenta el término Inteligencia Organizacional en la literatura especializada por Wilensky. Sin embargo, no es hasta 1992, en una Conferencia Internacional sobre Economía en Tokio, Japón, que Takehiko Matsuda presenta un artículo titulado "Inteligencia Organizacional: su importancia como proceso y como producto" que tuvo gran impacto en la comunidad científica. Actualmente, la 10 debe ser considerada como una capacidad institucional que permite desarrollar adecuados procesos cognitivos e informacionales, de forma que las organizaciones puedan, bajo un enfoque de aprendizaje continuo, percibir y analizar información de forma estratégica, generar conocimiento organizacional, $\mathrm{y}$ tomar decisiones efectivas para solucionar problemas y aprovechar oportunidades, tener un mejor desempeño organizacional y alcanzar un buen posicionamiento de manera más eficaz y eficiente.

Existe una polémica sobre el alcance de las concepciones de Inteligencia Organizacional, Inteligencia Empresarial, Inteligencia Corporativa e Inteligencia Competitiva, así como los puntos comunes y divergentes entre cada una de estas definiciones. Un conjunto de autores, apuntan que la inteligencia organizacional debe ser calificada como el concepto más general, al interpretar 
esta capacidad en el contexto de cualquier tipo de organizaciones. Tanto como proceso, como producto o como servicio la IO tiene en su núcleo a la información. Como proceso trabaja en la creación de productos (por ejemplo: informes de inteligencia de diverso tipo) que pueden ser hechos para prestar servicios al propio centro o a una entidad externa. Estos productos derivan en la importancia que tiene para las organizaciones que su flujo informacional sea ininterrumpido, transparente y controlado de manera que permita elegir las mejores opciones para solucionar problemas del ambiente interno o externo a la organización.

Al analizar los elementos teóricos, los modelos y la metodología de la IO se concluye que está formada por veinticinco componentes que se han agrupado en las siguientes categorías:

- Elementos cognitivos e informacionales

Capital Intelectual, Memoria organizacional, Capacidad para tomar decisiones, Percepción, Agregación de valor, Aprendizaje organizacional, Cognición organizacional, Innovación, Razonamiento organizacional, Sistema de Información, Procesos informacionales, Tecnologías de la Información y la Comunicación (TIC).

- Factores organizacionales que inciden en la 10

Cultura organizacional, Comunicación organizacional Estructura organizacional, Estrategia organizacional, Ambiente interno y externo, Clima organizacional, Relaciones sociales, Apoyo organizacional, Motivación y Recursos humanos.

La Inteligencia Organizacional está caracterizada por: Habilidades para la creación de valor, Creatividad, Ambiente dinámico, Reglas y rutinas para la toma de decisiones, Pragmatismo, Satisfacer de necesidades de los usuarios y clientes, Proyección estratégica, Dinamismo, Uso de fuentes públicas y privadas, Capacidad informacional, Reducción de riesgos, Innovación combinada, Aumentar la calidad, productividad y competitividad, Información filtrada, Instrumento cultural y Evolución continua. Las características de la IO van a ser estándares para las organizaciones inteligentes, aunque el desarrollo de ellas depende de las características propias de la organización (su situación económica, política, contextual, cultural, etc.) que van a determinar el grado de 
inteligencia que va a alcanzar.

Es indiscutible que para una institución de estos tiempos es de suma importancia alcanzar la condición de organización inteligente, por las facilidades que esto representa para el flujo de procesos dentro de la organización. Lo cierto es que, una de las aplicaciones de la 10 que más utilidad y beneficio trae a las organizaciones y sus directivos es la toma de decisiones acertadas. La precisión de saber cuál es la decisión correcta en el momento oportuno y en un contexto determinado, es posible solo para un tipo de organizaciones: las inteligentes; esas que han sido capaces de poner todos sus recursos humanos y tecnológicos en función de lograr un funcionamiento óptimo.

\section{REFERENCIAS}

AKGÜN, A. E.; BYRNE, J.; KESKIN, H. Organizational intelligence: a structuration view. Journal of Organizational Change Management, v. 20, n. 3, p. 272-289, 2007.

\section{ALABART-PINO, Y. Contribución al diagnóstico de la cultura} organizacional en el sector empresarial cubano. 2003. $98 \mathrm{~h}$. Tesis (Máter en Ciencias), Instituto Superior Politécnico José A. Echeverría, La Habana, Cuba. Disponible en:

http://scholar.google.es/scholar?q=Contribuci\%C3\%B3n+al+diagn\%C3\%B3stic o+de+la+cultura+organizacional, +en+el+sector+empresarial+cubano\&btnG=\&hl =es\&as_sdt=0,5. Recuperado el: 03 nov. 2015.

ALABART-PINO, Y. La Cultura y la Inteligencia Organizacional, pilares para la Innovación en el Siglo XXI. En: VALENTIM, M. L. P.; MÁS-BASNUEVO, A. (Ed.). Inteligência Organizacional. São Paulo: Cultura Acadêmica; Marília: Oficina Universitária, 2015. p. 293-312.

ALBRECHT, K. Organizational intelligence \& knowledge management: Thinking outside the silos. Executive white paper, 2002. Disponible en: http://www. KarlAlbrecht. com. Recuperado el: 03 nov. 2015.

$\mathrm{CHOO}, \mathrm{C}$. W. La organización inteligente: el empleo de la información para dar significado, crear conocimiento y tomar decisiones. Cuauthémoc: Oxford University Pres, 1999.

CRONQUIST, B. Organizational Intelligence: a dynamic knowledge creating activity embedded in organizational routines, 2004. 
CUBILLO, J. La inteligencia empresarial en las pequeñas y medianas empresas competitivas de América Latina: algunas reflexiones. Ciência da Informação, v. 26, n. 3, 1997.

ERFANIKHANGHAHIE, M; JAFARI, P. Organizational intelligence and its development in university. Aval Printing. Tehran. FarashenakhtiAndishe Press, 2010.

GONZÁLEZ, F. Comunicación e inteligencia organizacional como pilares de una gestión efectiva, 2015. Disponible en:

https://www.gestiopolis.com/comunicacion-e-inteligencia-organizacional-comopilares-de-una-gestion-efectiva. Recuperado el: 10 ago. 2015.

HABER-VEGA, A. Metodología para la introducción de un modelo de Inteligencia Organizacional en la Delegación del CITMA de La Habana. Tesis (Doctorado) - Universidad de La Habana, La Habana, 2015.

HABER-VEGA, A.; MÁS-BASNUEVO, A. Inteligencia Organizacional: conceptos, modelos y metodologías. Encontros Bibli: Revista Eletrônica de Biblioteconomia e Ciência da Informação, v. 18, n. 38, 2013.

HAECKEL, S.; NOLAN, R. Managing by Wire. Harvard Business Review, v. 71, n. 5, sep./oct. 1993.

HALAL, W. E. Organizational intelligence: What is it and how can manager use it to Improve Performance?. Knowledge Management Review, p. 20-25, abr. 1998.

KIANI, M. P.; POURJAFARIJOZAM, M.; POURJAFARIJOZAM, M.

Organizational Intelligence, survival factor of today's organizations. International Journal of Advanced Studies in Humanities and Social Science, v. 1, n. 5, p. 469-484, 2013.

LOZANO-OVIEDO, J.; GONZÁLEZ-CAMPO, C. H. Una propuesta para la definición de la inteligencia organizacional. Universidad \& Empresa, v. 16, n. 26, p. 159-175, 2013. Disponible en:

dx.doi.org/10.12804/rev.univ.empresa.26.2014.06. Recuperado el: 03 nov. 2015.

MÁS-BASNUEVO, A. Antecedentes y situación actual de los conceptos y métodos para el desarrollo de la inteligencia organizacional. Acimed, v. 13, n. 4, 2005a. Disponible en:

http_//bvs.sld.cu/revistas/aci/vol13_4_05/aci02405.htm. Recuperado el: 3 nov. $201 \overline{5}$.

MÁS-BASNUEVO, A. Modelo para la introducción de la Inteligencia Organizacional en la Delegación del CITMA en Holguín. 2005b. Tesis (Doctorado), Universidad de La Habana, La Habana, Cuba. 
MATSUDA, T. Organizational intelligence: its significance as a process and as a product. En: Proceedings of the International Conference on Economics/Management and Information Technology. Tokio: The Japan Society for Management Information, 1992. p. 219-222.

MCMASTER, M. Organizational Intelligence. 1996. Disponible en: http://www.parshift.com/speakers/speak011.htm. Recuperado el: 12 de nov. 2015.

MÜLLER, R.; CASTILHO J. N. C. Inteligência organizacional como ferramenta de gestao: um referencial teórico integrado. Revista Expectativa, v. 11, n. 11, p. 83-102, jan./dez. 2012.

MILLER, J. P. O milênio da inteligência competitiva. Porto Alegre: Bookman, 2002.

NÚÑEZ PAULA, I. Enfoque Teórico-Metodológico para la determinación de las necesidades que deben atender los sistemas de información en las organizaciones o comunidades. Tesis (Doctorado), Universidad de La Habana, La Habana, Cuba, 2002.

NORTH, K.; PÖSCHL, A. Un test de inteligencia para las organizaciones. Trujillo: La Coria, 2003. p. 183-192.

OBERSCHULTE, H. Organisatorische Intelligenz-ein Vorschlag zur Konzeptdifferenzierung. Management for schung, v. 6, p. 41-81, 1996.

OROZCO SILVA, E. La inteligencia organizacional en la industria biofarmacéutica. Ciência da informação, v. 28, n. 1, 1999.

OROZCO SILVA, E. El lugar de la inteligencia empresarial en el entorno conceptual de la gestión del conocimiento. Evolución en Cuba. El profesional de la información, v. 10, n. 7-8, p. 14-22, 2001.

OROZCO-SILVA, E. Inteligencia empresarial: Qué y cómo. La Habana: Biomundi, IDICT, 2009.

PONJUÁN DANTE, G. Gestión de Información en las organizaciones: principios, conceptos y aplicaciones. Santiago (Chile): CECAPI, 1998.

RODRÍGUEZ-CRUZ, Y. La Inteligencia Organizacional bajo un enfoque integrador de Gestión Documental, de Información y del Conocimiento. En: VALENTIM, M. L. P.; MÁS-BASNUEVO, A. (Ed.). Inteligência Organizacional. São Paulo: Cultura Académica; Marília: Oficina Universitária, 2015. p. 341-372.

SILVA, E. da. Informação e conhecimento: elementos essenciais para a geração de inovação. En: VALENTIM, M. L. P.; MÁS-BASNUEVO, A. (Ed.). Inteligência Organizacional. São Paulo: Cultura Acadêmica; Marília: Oficina Universitária, 2015. p. 249-270. 
TEIXEIRA, T. M.; VALENTIM, M. L. P. Informação como Insumo para a Inteligência Organizacional. En: VALENTIM, M. L. P.; MÁS-BASNUEVO, A. (Ed.). Inteligência Organizacional. São Paulo: Cultura Acadêmica; Marília: Oficina Universitária, 2015. p. 195-214.

TYSON, K. W. The Complete Guide to Competitive Intelligence: gathering, analyzing, and using competitive intelligence. Illinois: Kirk Tyson International Ltd., 1998.

WILENSKY, $\mathrm{H}$. Organizational intelligence: knowledge and policy in goverment and industry. New York: Basic Books; 1967.

YAGHOUBI, Nour-mohammad; GHOLAMI, S.; ARMESH, H. The relationship between strategic processes of knowledge management and organizational intelligence. African Journal of Business Management, v. 6, n. 7, p. 26262633, 2012.

\title{
INTELIGÊNCIA ORGANIZACIONAL: ABORDAGEM TEÓRICA À SUA CONCEPÇÃO E DESENVOLVIMENTO
}

\begin{abstract}
RESUMO
Introdução: Inteligência Organizacional (IO) é uma capacidade organizacional que suporta a tomada de decisão estratégica. No entanto, a diversidade de termos e práticas associados teve um impacto na aplicação de múltiplas abordagens com diferentes componentes, processos e fatores institucionais. Objetivo: Correspondentemente, este estudo tem como objetivo identificar e esclarecer as bases teóricas e metodológicas que influenciaram o desenvolvimento das OI e seus processos particulares. Metodologia: A análise documental é utilizada como principal método de pesquisa, e a análise de conteúdo permite identificar os princípios e abordagens teóricas que caracterizaram essa capacidade. Resultados: A partir da análise de modelos e metodologias de OI, são identificados os componentes específicos dessa capacidade, sua articulação com os processos de desenvolvimento específicos: Vigilância Tecnológica, Inteligência Competitiva, Business Intelligence, Monitoramento da Informação, bem como os diversos elementos e metodologias, factores a ter em conta para o seu desenvolvimento nas organizações. Conclusões: A 10 constitui uma capacidade organizacional que se desenvolve a partir de um conjunto de processos específicos e elementos particulares que, sem sua articulação adequada, não conseguem obter vantagens estratégicas e processos precisos de decisão organizacional.
\end{abstract}

Descritores: Inteligência Organizacional. Informação. Conhecimento. Inteligência. Organizações.

\section{ORGANIZATIONAL INTELLIGENCE: THEORETICAL APPROACH TO ITS CONCEPTION AND CENTRALIZED DEVELOPMENT}




\begin{abstract}
Introduction: Organizational Intelligence $(\mathrm{IO})$ is an organizational capacity that supports strategic decision-making. However, the diversity of associated terms and practices has had an impact on the application of multiple approaches with different components, processes and institutional factors. Objective: Correspondingly, this study aims to identify and clarify the theoretical and methodological bases that have influenced the development of the 10 and its particular processes. Methodology: Documentary Analysis is used as the main research methods, and the content analysis allows identifying the theoretical principles and approaches that have characterized this capacity. Results: From the analysis of 10 models and methodologies, the specific components of this capacity are identified, their articulation with the particular development processes: Technological Surveillance, Competitive Intelligence, Business Intelligence, Information Monitoring, as well as the various elements and factors to take into account for their development in organizations. Conclusions: The IO constitutes an organizational capacity that is developed from a set of specific processes and particular elements that without their adequate articulation, cannot obtain strategic advantages and accurate organizational decision processes.
\end{abstract}

Descriptors: Organizational intelligence. Information. Knowledge. Intelligence. Organizations. 